\title{
Review
}

\section{CPT Symmetry and Its Violation}

\author{
Ralf Lehnert ${ }^{1,2}$ \\ 1 Indiana University Center for Spacetime Symmetries, Bloomington, IN 47405, USA; ralehner@indiana.edu \\ 2 Leibniz Universität Hannover, Welfengarten 1, Hannover 30167, Germany \\ Academic Editor: Eberhard Widmann \\ Received: 2 September 2016; Accepted: 12 October 2016; Published: 28 October 2016
}

\begin{abstract}
One of the most fundamental symmetries in physics is CPT invariance. This article reviews the conditions under which CPT symmetry holds by recalling two proofs of the CPT theorem: The original Lagrangian-based analysis and the more rigorous one in the context of axiomatic quantum field theory. The presentation of the proofs is followed by a discussion of the major physical implications that arise from CPT symmetry. Motivated by recent theoretical and experimental interest in CPT tests, various approaches to the violation of CPT symmetry are mentioned, and it is briefly discussed how they evade the CPT theorem. An attempt has been made to keep this work self-contained and at a level suitable for a wider readership by excising as many technical aspects as possible.
\end{abstract}

Keywords: CPT theorem; implications of CPT symmetry; CPT-symmetry violation

\section{Introduction}

In the last century, the concept of symmetry in the laws of nature has played an increasingly important role. On the one hand, symmetries are often desirable for reasons of theoretical consistency, for their aesthetic appeal, as guiding principles for approaches to new physics, etc. On the other hand, scientific progress has also been achieved in the past by the notion of approximate symmetries and by the idea of symmetry violation.

In our present understanding, spacetime-although dynamical itself-serves as the arena for all physical events. Spacetime symmetries therefore possess a particularly fundamental character in physics. The present article focuses on the case of a flat Minkowski spacetime, i.e., situations in which gravity can be neglected. The known continuous spacetime symmetries in this situation are global translations in both space and time as well as global Lorentz transformations, which include both boosts and rotations. The established discrete spacetime symmetry is given by CPT invariance, where $\mathrm{CPT}$ represents the combined transformation of charge conjugation $\mathrm{C}$, parity inversion $\mathrm{P}$, and time reversal T. It turns out that Lorentz and CPT symmetry are closely intertwined, a fact with significant consequences for experimental investigations of spacetime symmetries. The purpose of the current work is to review some theoretical aspects of this intimate relation with as few technical details as possible and with the goal of broad accessibility.

Loosely speaking, $\mathrm{C}$ can be thought of as exchanging particle and antiparticle, $\mathrm{P}$ as reflecting all three spatial coordinates (e.g., $\vec{r} \rightarrow-\vec{r}$ ), and $\mathrm{T}$ as reversing time coordinates (e.g., $t \rightarrow-t$ ). However, we remind the reader that special care is required, for example, in cases involving pseudovectors, such as the angular momentum $\vec{L}=\vec{r} \times \vec{p}$. Although some physical theories, like quantum electrodynamics, are separately invariant under $\mathrm{C}, \mathrm{P}$ and $\mathrm{T}$, this is not the case for the entire body of established physics. In fact, $\mathrm{CPT}$ is the sole combination of $\mathrm{C}, \mathrm{P}$ and $\mathrm{T}$ observed as an exact symmetry of nature at the level of known fundamental physics. We mention that in nonrelativistic physics, antiparticles need to be introduced to some extent by hand. In the context of combining special relativity and quantum mechanics, however, the existence of antiparticles is essentially required. In this sense, the setting 
of relativistic quantum theory is most natural and meaningful for charge-conjugation symmetry or its violation.

When interpreting and investigating the symmetry properties of a model, it is critical to consider the correct type of transformation. Coordinate transformations are typically unsuitable in such contexts. In physics, coordinates provide an elegant mathematical tool for the description of observables and relations between them. However, they merely represent a labeling of spacetime points, which is to a large extent arbitrary and without intrinsic physical relevance. In other words, physics should be independent of the chosen coordinate system and independent of the observer. For this reason, coordinate independence is also called observer invariance in the literature. On the other hand, studying as to whether the transformed dynamical variables continue to satisfy the same equation is more meaningful. Note that this corresponds to the actual experimental situation, in which measurements before and after the transformation of the apparatus (and not the observer) are compared. Such types of physical transformations are often called particle transformations. In the present context, this means a CPT transformation on the coordinate system is insufficient to study the CPT properties of a given model. Instead, the transformations need to be set up such that they correspond to transforming the experimental set-up.

Motivations to study CPT invariance are threefold. First, the CPT theorem illustrates that CPT symmetry is based only on mild physical requirements (conventional quantum mechanics, Lorentz symmetry, energy positivity, and causality) which underlines its truly foundational character. As such, tests of CPT invariance are at the same time probes of various cornerstones of physics. Second, CPT contains charge conjugation, and therefore represents a symmetry between matter and antimatter. However, cosmological observations indicate that the universe is dominated by matter suggesting an asymmetry between matter and antimatter in nature. Although this asymmetry can be explained in principle with the three Sakharov conditions [1] of C and CP violation, Baryon-number violation, and interactions out of thermal equilibrium, the amount of $\mathrm{CP}$ violation contained in the Standard Model appears to be insufficient for a convincing explanation of the observed baryon asymmetry. On the other hand, Planck-size CPT violation may evade the Sakharov conditions and generate a significant overabundance of matter [2]. Third, some theoretical approaches to physics beyond the Standard Model, such as those mentioned in Section 4, contain a violation of CPT invariance as a key prediction. For these reasons, CPT tests represent an interesting and timely avenue for experimental research in fundamental physics.

This work contains two simplified versions of the proof of the CPT theorem with the goal to bring to light clearly the minimal physical requirements for CPT symmetry to hold. The proofs are followed by a discussion of three types of physical consequences that can be inferred from CPT invariance. Based on the exposed ingredients for the CPT theorem, the present article proceeds to give a brief account of the implications of, and possible mechanisms for, violations of CPT symmetry. Throughout, we work in natural units $\hbar=c=1$, and our metric signature is $\eta^{\mu v}=\operatorname{diag}(+,-,-,-)$.

\section{The CPT Theorem}

The CPT theorem states that under mild technical assumptions any unitary, local, Lorentzinvariant point-particle quantum field theory in flat Minkowski space is CPT invariant. This theorem was first established in the early 1950s in the context of Lagrangian field theory. A few years later, a general version of the theorem was proved within the more abstract and mathematically rigorous framework of axiomatic field theory. In the following two subsections, the line of reasoning behind each of the two versions of the proof will be reviewed with particular focus on the key ingredients on which the proofs rely.

There is a certain degree of arbitrariness in the construction of $\mathrm{C}, \mathrm{P}$, and $\mathrm{T}$ that cannot be removed entirely from their definition without $a d$ hoc assumptions. An example is given by phase factors that leave unchanged the product CPT. Instead, one can consider a suitable discrete reflection $\Theta$ and 
establish the invariance of quantum field theories under this reflection. Indeed, some authors proceed in exactly this way. However, the decomposition of $\Theta$ into $C, P$, and T,

$$
\Theta=C P T,
$$

does provide a clear physical interpretation of this reflection operation. The order in which the individual C, $\mathrm{P}$, and $\mathrm{T}$ transformations are performed turns out to be physically irrelevant.

Adopting the usual mathematical practice, we initially set up $\Theta$ to transform a physical state $|\psi\rangle$ to its CPT-conjugate state $|\bar{\psi}\rangle$ in the same physical Hilbert space:

$$
|\Theta \psi\rangle=\Theta|\psi\rangle \equiv|\bar{\psi}\rangle .
$$

Note that we consider a single theory that contains both states $|\psi\rangle$ and $|\bar{\psi}\rangle$. Since $\Theta$ involves charge conjugation, which connects particles and antiparticles, this means we take the theory to describe both particle and antiparticle states at the same time. In particular, we do not consider situations such as a mapping of the Pauli equation for an electron to the Pauli equation for a positron: These are two distinct models, each with their own parameters that can in principle be specified separately. The property

$$
\Theta \Theta=1 \quad \Rightarrow \quad \Theta=\Theta^{-1}=\Theta^{\dagger}
$$

seems reasonable on physical grounds: two charge conjugations, two parity reflections, and two time reversals should leave the physics unaffected. In the last step, we have used the fact that quantum symmetry operations are known to be (anti)unitary. We finally remark that for the general form of the transformation law for quantum operators $\mathcal{A}$, we may take

$$
\mathcal{A} \rightarrow \mathcal{A}_{\mathrm{CPT}}=\Theta \mathcal{A} \Theta^{\dagger}=\Theta^{\dagger} \mathcal{A} \Theta .
$$

The second equality holds on account of Equation (3).

\subsection{Proof Based on Lagrangian Field Theory}

The first approaches to the CPT theorem by Bell [3], Lüders [4,5], and Pauli [6], and implicitly already by Schwinger [7], are to a large extent based on the Lagrangian formalism in quantum field theory. These approaches proceed essentially by construction: all physically acceptable terms that can enter a Lagrangian density are formed. Alternatively, some of these authors work at the level of the interaction Hamiltonian or the actual field equations. In any case, it is then shown that each of these terms must necessarily be CPT symmetric. Often, this procedure is illustrated explicitly by focusing on the physically most relevant cases of a scalar particle, a spin- $\frac{1}{2}$ fermion, and a spin- 1 boson: All known elementary particles fall into one of these categories.

Proceeding in this manner, we need the properties of the field operators under the reflection $\Theta$, i.e., under the CPT transformation:

$$
\begin{aligned}
\phi(x) & \rightarrow \Theta \phi(x) \Theta^{\dagger}=\phi^{\dagger}(-x), \\
\psi(x) & \rightarrow \Theta \psi(x) \Theta^{\dagger}=-\gamma_{5} \psi^{+T}(-x), \\
A_{\mu}(x) & \rightarrow \Theta A_{\mu}(x) \Theta^{\dagger}=-A_{\mu}^{\dagger}(-x),
\end{aligned}
$$

where we have adopted the gamma-matrix conventions of Ref. [8]. Here, the transpose operation denoted by the superscript $T$ is not to be confused with the time-reversal transformation; it applies only to the spinor indices, and does not refer to the entire quantum-field Hilbert space. We mention that by virtue of Equation (3), the CPT operator $\Theta$ and its Hermitian conjugate may be interchanged in Equations (5)-(7) in much the same way as in Equation (4). 
The demonstration that $\Theta$ can indeed be interpreted as the CPT-transformation operator is most convincingly achieved in quantum field theory. Here, we merely motivate with a few heuristic remarks that Equations (5)-(7) indeed implement CPT conjugation. The inversion of the spacetime point $x \rightarrow-x$ is intuitively reasonable. It arises from a PT reflection, and $\mathrm{C}$ would be expected to leave $x$ unchanged. The Hermitian or complex conjugation of the field operators arises from $C$. Consider, for example, an electrically charged scalar field $\phi$ with charge $q$. It must couple to the photon field $A_{\mu}$ via a gauge-covariant derivative $D_{\mu}=\partial_{\mu}-i q A_{\mu}$ if the usual gauge invariance and the resultant charge conservation is to be maintained. The field equation for $\phi^{\dagger}$ then contains $D_{\mu}^{*}=\partial_{\mu}+i q A_{\mu}$ because it can be obtained via Hermitian conjugation from the field equation for $\phi$ [9]. Inspection of $D_{\mu}$ and $D_{\mu}^{*}$ reveals that $\phi$ and $\phi^{\dagger}$ couple with opposite charge $q$ to the electromagnetic field compatible with being charge conjugates of one another. The minus sign in the transformation of $A_{\mu}$ is also intuitively reasonable by looking at the example of classical electromagnetism. Changing the sign of source charges $(\rho, \vec{j}) \stackrel{\mathrm{C}}{\longrightarrow}(-\rho,-\vec{j})$, parity inversion $(-\rho,-\vec{j}) \stackrel{\mathrm{P}}{\longrightarrow}(-\rho,+\vec{j})$, and time reversal $(-\rho,+\vec{j}) \stackrel{\mathrm{T}}{\longrightarrow}(-\rho,-\vec{j})$ changes the overall sign of the source current $j_{\mu}$, so the sign of $A_{\mu}$ must reverse as well [10].

Another ingredient in the construction of Lagrangians is the 4-gradient $\partial_{\mu}$. This operator remains unchanged under $\Theta$ :

$$
\partial_{\mu} \rightarrow \Theta \partial_{\mu} \Theta^{\dagger}=\partial_{\mu}
$$

This behavior stems from the question of whether equations of motion of the same form, and in particular with the same derivative structure, are satisfied by the CPT conjugate fields. For example, consider $\frac{\partial}{\partial x^{\mu}} A^{\mu}(x)=c$ for real-valued $A^{\mu}(x)$ and $c=$ const. CPT symmetry holds if $\Theta A^{\mu}(x) \Theta^{+}=A^{\mu}(-x)$ also satisfies this equation, i.e., $\frac{\partial}{\partial x^{\mu}} A^{\mu}(-x)=c$. The equation $-\frac{\partial}{\partial x^{\mu}} A^{\mu}(-x)=c$ holds trivially because it follows from the original equation by renaming $x \rightarrow-x$. This is consistent with the aforementioned fact that pure changes of coordinates should not have physical effects.

For general, dynamical rank- $n$ tensor densities $\mathcal{T}^{\mu_{1} \mu_{2} \ldots \mu_{n}}(x)$, the following relation holds:

$$
\Theta \mathcal{T}_{\mu_{1} \mu_{2} \ldots \mu_{n}}(x) \Theta^{\dagger}=(-1)^{n} \mathcal{T}_{\mu_{1} \mu_{2} \ldots \mu_{n}}^{\dagger}(-x) .
$$

Roughly speaking, tensor densities with an even (odd) number of indices are CPT even (odd). Note that this property is compatible with the above definitions (5), (7), and (8). For example, the transformation of the electromagnetic field-strength tensor $F^{\mu v}(x)=\partial^{\mu} A^{v}(x)-\partial^{v} A^{\mu}(x)$ can either be obtained with Equation (9) for $F^{\mu v}(x)$ or with Equations (7) and (8) for $\partial^{\mu} A^{v}(x)-\partial^{v} A^{\mu}(x)$, and the results agree:

$$
\begin{aligned}
\Theta F^{\mu v}(x) \Theta^{\dagger} & =\Theta\left[\partial^{\mu} A^{v}(x)-\partial^{v} A^{\mu}(x)\right] \Theta^{\dagger} \\
& =-\frac{\partial}{\partial x_{\mu}} A^{v}(-x)+\frac{\partial}{\partial x_{v}} A^{\mu}(-x) \\
& =\frac{\partial}{\partial\left(-x_{\mu}\right)} A^{v}(-x)-\frac{\partial}{\partial\left(-x_{v}\right)} A^{\mu}(-x) \\
& =+F^{\mu v}(-x) .
\end{aligned}
$$

Using the spin-statistics theorem, we will argue below that spinors are also compatible with the transformation law (9). Nondynamical objects with Lorentz indices do not follow this rule. For example, a complex-valued four-vector $z^{\mu}$ transforms as four complex numbers $z^{\mu} \rightarrow \Theta z^{\mu} \Theta^{\dagger}=z^{\mu *}$ and involves complex conjugation according to Equation (11) to be discussed next.

Antilinearity of $\mathbf{T}$. The time-reversal transformation is an antilinear operation [11]; it complex conjugates complex numbers $z$ : $T z T^{-1}=z^{*}$. The other two transformations, charge conjugation $C$ and parity inversion $P$, are both linear. This means that $\Theta$, just like $T$, is also antilinear:

$$
z \rightarrow \Theta z \Theta^{\dagger}=z^{*}
$$


This implies in particular that Lagrangian parameters, such as couplings, masses, mixing angles, entries of Dirac matrices, etc. are complex conjugated under the CPT transformation. We remark that $\Theta^{-1}=\Theta^{\dagger}$, so that $\Theta \Theta^{+}=\Theta^{\dagger} \Theta=1$. Antilinear operators that obey this additional condition are called antiunitary.

The action of $\Theta$ and other antiunitary operators on matrix elements needs to be defined carefully, so for the moment we switch from the bra-ket notation to one that better clarifies how operators act on states $\langle\psi|\mathcal{A}| \chi\rangle=(\psi, \mathcal{A} \chi)=\left(\mathcal{A}^{\dagger} \psi, \chi\right)$. Consider, for example, an ordinary unitary transformation $U$ on both the states $\psi$ and $\chi$ as well as on the operator $\mathcal{A}:(\psi, \mathcal{A} \chi) \rightarrow\left(\psi^{\prime}, \mathcal{A}^{\prime} \chi^{\prime}\right)=\left(U \psi, U \mathcal{A} U^{\dagger} U \chi\right)=$ $\left(\psi, U^{\dagger} U \mathcal{A} U^{\dagger} U \chi\right)=(\psi, \mathcal{A} \chi)$, which leaves the matrix element trivially unchanged. For our present purpose of searching for physical invariances, such trivial types of transformation are uninteresting. Instead, a nontrivial unitary or antiunitary transformation of a matrix element involves the states only $(\psi, \mathcal{A} \chi) \rightarrow\left(\psi^{\prime}, \mathcal{A} \chi^{\prime}\right)$ consistent with the mathematical definition of a transformation on a Hilbert space. For an antiunitary transformation, this yields

$$
\left(\psi^{\prime}, \mathcal{A} \chi^{\prime}\right)=(\Theta \psi, \mathcal{A} \Theta \chi)=(\psi, \underbrace{\Theta^{\dagger} \mathcal{A} \Theta}_{\mathcal{A}_{\mathrm{CPT}}} \chi)^{*}=\left(\chi, \mathcal{A}_{\mathrm{CPT}}^{+} \psi\right) .
$$

Here, $\mathcal{A}_{\mathrm{CPT}}$ is the CPT-conjugate operator determined by the general rules above. The complex conjugation of the matrix element in the second step differs from the usual unitary-operator case; it follows from the mathematical theory of antilinear operators. The last step uses the usual properties of Hilbert-space scalar products. Physical observables are usually Hermitian. Moreover, they may describe a process in time, such as scattering $\langle f|\mathcal{S}| i\rangle$, with an initial and final state. The last step in Equation (12) then reads $\langle f|\mathcal{S}| i\rangle \stackrel{\mathrm{CPT}}{\longrightarrow}\left\langle i\left|\mathcal{S}_{\mathrm{CPT}}\right| f\right\rangle$, i.e., CPT affects not only the scattering operator $\mathcal{S}$, but also interchanges initial and final states, as expected from the time-reversal factor in CPT.

Connection between Spin and Statistics. The proof of the CPT theorem proceeds by using the connection between spin and statistics in the following way [12]. Since the Lagrangian density is a Lorentz scalar, spinor indices must be contracted, so that spinors always come in pairs. These spinor bilinears are formed such that they transform like Lorentz tensors, i.e., that they possess zero, one, or two Lorentz indices: $\bar{\psi} \psi, \bar{\chi} \gamma_{5} \gamma^{\mu} \psi, \bar{\psi} \sigma^{\mu v} \psi$, etc. This allows a straightforward Lorentz-invariant coupling to other tensors, like $A_{\mu}, \phi$, and $\partial_{\mu}$. A CPT transformation yields $\bar{\chi}(x) \psi(x) \rightarrow$ $\Theta \bar{\chi}(x) \Theta^{\dagger} \Theta \psi(x) \Theta^{\dagger}=\chi^{T}(-x) \gamma_{5} \gamma^{0 *} \gamma_{5} \psi^{+T}(-x)=-\chi^{T}(-x) \gamma^{0 *} \psi^{+T}(-x)=-\chi^{\dagger T+} \gamma^{0 *} \psi^{+T}(-x)=$ $-\left[\psi^{T}(-x) \gamma^{0 T} \chi^{T \dagger}(-x)\right]^{\dagger}=+\left[\left\{\chi^{\dagger}(-x) \gamma^{0} \psi(-x)\right\}^{T}\right]^{\dagger}$. Here, we used that $\gamma^{0}$ and $\gamma_{5}$ anticommute, and that $\gamma_{5} \gamma_{5}=1$. The crucial step is the last one, in which we simplified the spinor-space transpose; it requires reversing the order of $\chi$ and $\psi$. It is this step that uses the connection between spin and statistics: fermion fields anticommute. The term in the curly brackets is a spinor-space scalar, so the spinor transposition may be left out and we have $\bar{\chi}(x) \psi(x) \rightarrow[\bar{\chi}(-x) \psi(-x)]^{\dagger}$. Extension of this reasoning including fermion anticommutation to the other Dirac bilinears shows that they also follow the general rule (9) established above for dynamical tensor densities.

Lorentz invariance. We have already used Lorentz symmetry implicitly in the above individual ingredients for the construction of field-theory Lagrangian densities: the Minkowski position $x$, the scalar, spinor, and vector fields in Equations (5)-(8), and the general tensor densities in Equation (9) are all realizations of the Lorentz group, i.e., rotations and boosts of these objects are implemented by Lorentz transformations. In Lagrangian field theory in Minkowski space, Lorentz invariance is guaranteed if the action, and thus the Lagrangian density, are both Lorentz scalars. This implies that all fields and derivatives in a Lagrangian density must be combined such that not only the spinor indices, but also all Lorentz indices are properly contracted. Through this pairwise contraction, the total number of Lorentz indices in each Lagrangian term of field products must be even $n=2 k, k \in \mathbb{N}$. According to Equation (9), this yields for the Lagrangian density

$$
\Theta \mathcal{L}(x) \Theta^{\dagger}=(-1)^{2 k} \mathcal{L}^{\dagger}(-x)=\mathcal{L}^{\dagger}(-x)
$$


under CPT. Here, the assumption has been made that all fields in $\mathcal{L}(x)$ are combined at the same spacetime point $x$. We will have to say more about such pointlike interactions below [13].

Unitarity. The next ingredient for the CPT theorem is a Hermitian Lagrangian density $\mathcal{L}=\mathcal{L}^{+}$, so that with Equation (13), we have

$$
\Theta \mathcal{L}(x) \Theta^{\dagger}=\mathcal{L}(-x)
$$

A Hermitian $\mathcal{L}$ will lead to a Hermitian Hamiltonian $H$. The time evolution $U(t)=\exp (-i H t)$ is then unitary, so that probability is conserved in time: $\left\langle t_{0}+t \mid t_{0}+t\right\rangle=\left\langle t_{0}\left|U^{\dagger}(t) U(t)\right| t_{0}\right\rangle=\left\langle t_{0} \mid t_{0}\right\rangle$. Without this property, a conventional interpretation of the quantum mechanics of closed systems would appear to be difficult.

Point interactions. To conclude the Lagrangian version of the proof of the CPT theorem, we show that the action $S=\int d^{4} x \mathcal{L}(x)$ remains invariant under $\Theta$. With the above result (14), we write

$$
\Theta S \Theta^{\dagger}=\int d^{4} x \mathcal{L}(-x)=\int d^{4} y \mathcal{L}(y)=S,
$$

where we have changed the dummy integration variable $x \rightarrow-y$ in the second step. As mentioned earlier, the validity of this step hinges crucially upon the absence of non-pointlike interactions. Consider, for example, Lagrangian contributions of the form $\frac{1}{2} \partial^{\mu} \phi(x) \partial_{\mu} \phi(x)+\phi(x) \varphi(x-d)$ for two real scalar fields $\phi$ and $\varphi ; d^{\mu}$ is a constant nonzero four-vector. The equations of motion for $\phi$ read $\partial^{\mu} \partial_{\mu} \phi(x)=\varphi(x-d)$. This shows that the behavior of $\phi$ at $x$ is determined by the value of $\varphi$ at the point $x-d$ indicating an interaction at a distance. According to Equation (5), real scalars reverse the sign of their entire spacetime argument under CPT, i.e., $\Theta \phi(x) \Theta^{+}=\phi(-x)$ and $\Theta \varphi(x-d) \Theta^{+}=\phi(-x+d)$. The corresponding piece of the action therefore obeys $\Theta \delta S \Theta^{\dagger}=\int d^{4} x \Theta^{\dagger} \phi(x) \varphi(x-d) \Theta=$ $\int d^{4} x \phi(-x) \varphi(-x+d)=\int d^{4} y \phi(y) \varphi(y+d) \neq \delta S$. It thus becomes apparent that CPT symmetry is not automatically satisfied in theories with interactions at a distance. We remark that such interactions would typically be associated with violations of causality. The axiomatic proof the CPT theorem to be discussed next further illuminates the role of microscopic causality.

\subsection{Proof Based on Axiomatic Field Theory}

In 1950s, efforts to place quantum field theory on a more rigorous mathematical footing intensified. Within this mathematical-physics context, the Lagrangian formalism turned out to be too narrow, and sets of axioms, such as the Wightman axioms, were adopted [14]. A next natural step was then to ask what properties of quantum field theory follow rigorously from these axioms. For the case of CPT symmetry, this question was answered by Jost [15] in 1957. Although more technical, his proof of the CPT theorem thoroughly illuminates the close connection between Lorentz and CPT symmetry.

The core of the argument involves the complexified version of Lorentz transformations, which are essentially boosts and rotations by complex-valued velocities and angles acting on complex-valued Minkowski vectors, tensors, etc. When in the conventional real Lorentz-transformation laws complex velocities and angles are entered, physical interpretation is usually lost for most input values, but the equation may still be mathematically correct. However, it turns out that for judiciously chosen imaginary boosts and real rotation angles, a complete spacetime inversion, which goes a long way to a full CPT transformation, can be achieved. Now, this is clearly not a proper orthochronous Lorentz transformation. However, this transformation does have a physical interpretation and is usually already contained in the mathematical structure of the transformation law. It is this complexification feature of the Lorentz transformations in quantum physics that is used to prove the CPT theorem and that exposes the intimate connection between CPT and Lorentz symmetry.

Let us illustrate this idea with an example. It has already been argued above that the Minkowski position $x^{\mu}$ would change sign under CPT: $\Theta^{\dagger} x^{\mu} \Theta=-x^{\mu}$, i.e., CPT can be implemented by multiplying $x^{\mu}$ with $-\mathbb{1}$, where $\mathbb{1}$ is the $4 \times 4$ identity matrix. Consider a Lorentz transformation 
$\Lambda(w, \alpha)$ consisting of a boost along the $x$-axis with rapidity $w=\operatorname{arctanh} v$ and a rotation about the $x$-axis by an angle $\alpha$ :

$$
\Lambda(w, \alpha)=\left(\begin{array}{cccc}
\cosh w & \sinh w & 0 & 0 \\
\sinh w & \cosh w & 0 & 0 \\
0 & 0 & \cos \alpha & -\sin \alpha \\
0 & 0 & \sin \alpha & \cos \alpha
\end{array}\right)
$$

It is apparent that for any proper Lorentz transformation, which has velocities $-1<v<+1$ and thus rapidities $-\infty<w<+\infty$, and which has angles $0 \leq \alpha<2 \pi$, we have $\Lambda(w, \alpha) \neq-\mathbb{1}$. However, if we chose a purely imaginary $w \rightarrow \pi i$ and let $\alpha \rightarrow \pi$, we do obtain

$$
\Lambda(w=\pi i, \alpha=\pi)=-\mathbb{1} .
$$

This illustrates that if the equations of physics remain invariant not only for real-valued boosts and rotations, but also for complex-valued Lorentz transformations, we can expect them to be left unchanged also under spacetime inversion. In particular, spacetime inversion amounts to a CPT transformation for, e.g., spacetime points $x^{\mu}$.

The actual proof of the CPT theorem includes further physical input, such as energy positivity and microscopic causality. It also includes some technical mathematical aspects including an analysis of the circumstances under which the above analytic continuation into the complex plane is valid. The goal of the following paragraphs is to shed some more light on this proof.

Jost's proof proceeds in the context of Wightman's approach to rigorous quantum field theory in a flat-spacetime background [16]. Weak gravitational fields can be expanded about flat backgrounds and are in this sense included in the framework. Strong gravitational fields, however, seem to lie outside Wightman's rigorous quantum field theory, so that Jost's proof would need to be generalized.

Wightman's approach is based on a set of axioms that define what is meant by a sensible quantum field theory. To appreciate the generality of the CPT theorem, it is useful to spell out these axioms and comment on their physical significance. In the literature, a few variations of Wightman's definition of a quantum field theory can be found. For example, certain axioms may be combined into a single one or, vice versa, axioms may be separated into subaxioms. Nevertheless, in one form or another, the following physical assumptions are made:

(1) Lorentz- and translation-covariant Hilbert space $\mathbb{H}$. This assumption essentially states that we consider a relativistic version of quantum theory in which the usual rules of quantum mechanics apply. In particular, there are unitary operators $U(\Lambda, a)$ that implement Lorentz transformations $\Lambda$ and spacetime translations $a$. The unitarity of these transformations ensures that under $\Lambda$ and $a$ states in $\mathbb{H}$ transform to other states in $\mathbb{H}$ such that all transition amplitudes remain unchanged.

(2) Vacuum state. The Hilbert space contains a unique state, called the vacuum $|0\rangle$, that remains invariant under both the Lorentz transformations and the translations up to a phase $U(\Lambda, a)|0\rangle \sim|0\rangle$. In particular, the vacuum can neither have a nonzero four-momentum nor a nonzero angular momentum, as these quantities would change under $U(\Lambda, a)$. Together with axiom (4) below, the vacuum needs to be the state with lowest energy. These requirements are intuitively reasonable: the flat-spacetime vacuum looks the same to all inertial observers. An additional, more technical assumption is that $|0\rangle$ be cyclic. This essentially means that all other physical states in $\mathbb{H}$ can be constructed by acting with the field operators of axiom (3) on the vacuum. This property is akin to that of the usual quantum harmonic oscillator, where excited states can be reached from the ground state with the creation operator.

(3) Field operators. Physical quantities are represented by polynomials of field operators $\phi(x)$ acting on this Hilbert space. These field operators transform under the Lorentz transformations as scalars, vectors, tensors, spinors, etc. Moreover, these fields are set up such that each corresponds to a definite finite spin and mass allowing the usual particle interpretation. It turns out that field operators are mathematically not well-defined at a spacetime point, so there is the 
technical assumption of them being tempered distributions "smeared out" with test functions. This assumption can, for example, be used to establish continuity properties as the field operators vary with spacetime, but otherwise this level of rigor will be unnecessary for our present purposes.

(4) Energy positivity. Translation invariance leads to a conserved four-momentum operator $P^{\mu}$. Its zeroth component $P^{0}$, the energy operator or Hamiltonian, is postulated to have non-negative eigenvalues $p^{0} \geq 0$. Together with the condition of Lorentz symmetry, this implies that the four-momentum eigenvalues $p^{\mu}$ are lightlike or timelike four-vectors $p^{\mu} p_{\mu} \geq 0$. In other words, $p^{\mu}$ must lie in the forward momentum-space lightcone. This property is closely tied to the requirement of stability: if there were no lowest-energy state, it would seem difficult to prevent the system from filling an infinite number of pairs of positive- and negative-energy states.

(5) Microscopic causality. Many textbooks seems to suggest that the property of causality is automatically contained in a Lorentz-symmetric theory. However, consider a model with spacelike particle four-velocities $u^{\mu}$. Being a four-vector, $u^{\mu}$ transforms covariantly under the Lorentz transformations compatible with Lorentz symmetry. However, a spacelike four-velocity is associated with superluminal particle speeds and thus acausalities. For this reason, causality is imposed separately as follows. Field operators $\phi$ commute or anticommute if they cannot be connected by light signals: $[\phi(x), \phi(y)]_{ \pm}=0$ for $\left(x^{\mu}-y^{\mu}\right)\left(x_{\mu}-y_{\mu}\right)<0$. In the mathematical-physics literature, this requirement is sometimes also called locality. The microcauslity condition may be understood intuitively by recalling the usual quantum-mechanical uncertainty relation $\Delta_{\psi} A \Delta_{\psi} B \geq \frac{1}{2}|\langle\psi|[A, B]| \psi\rangle|$ for two Hermitian operators $A$ and $B$, with the usual definition of the uncertainty $\Delta_{\psi} \mathcal{A}=\sqrt{\left\langle\psi\left|\mathcal{A}^{2}\right| \psi\right\rangle-\langle\psi|\mathcal{A}| \psi\rangle^{2}}$ for any Hermitian operator $\mathcal{A}$ with respect to the state $|\psi\rangle$. Here, one measurement generally affects the other measurement because their uncertainties are not independent unless the commutator $[A, B]$ vanishes. A careful reasoning in the present context shows that with the above microcausality condition, the physics at $x$ cannot affect the physics at $y$ and vice versa if the separation between $x$ and $y$ is spacelike [17]. We remark in passing that in this axiomatic framework the spin-statistics theorem follows rigorously, so that the above choice between commutators and anticommutators is actually fixed: commutators for integer-spin fields and anticommutators for half-integer spin fields.

Note the generality of the above axioms. In particular, no Lagrangian is required, and the details of the field equations are not specified. This set of axioms is sufficient to prove CPT symmetry.

The basic field-theory objects in Wightman's approach are Wightman functions $\mathcal{W}$ defined simply as vacuum expectation values of field operators:

$$
\mathcal{W}\left(\Delta x_{1}, \Delta x_{2}, \ldots, \Delta x_{n}\right) \equiv\left\langle 0\left|\phi\left(x_{0}\right) \phi\left(x_{1}\right) \ldots \phi\left(x_{n}\right)\right| 0\right\rangle, \Delta x_{k} \equiv x_{k}-x_{k-1} .
$$

Here, the spacetime points $x_{k}$ are physical, i.e., each point is described by a set of four real numbers. The fact that the Wightman functions depend only on spacetime differences follows from translation invariance postulated in Axiom (1). The field operators can be of any type, i.e., scalar, vectors, tensor, spinor, etc. Now, the Wightman reconstruction theorem [18] roughly states that a quantum field theory is uniquely determined by its Wightman functions. The theorem follows from the axioms above. The significance of the reconstruction theorem in the present context is that the CPT theorem needs to be proved only for all the $\mathcal{W}$ : if they satisfy CPT symmetry, so will the corresponding quantum field theory. This simplifies matters because instead of working with abstract field operator and Hilbert-space states, one needs to consider ordinary functions only.

In what follows, our sole focus will be on scalar fields, which is sufficient to gain a flavor of the proof and to appreciate the significance of the physical ingredients needed. More general types of fields are considered in Ref. [16]. We first state the CPT theorem, and then proceed to prove it. Translated into the present context, the theorem asserts that the CPT-transformed Wightman functions 
should be identical to the original ones. As the Wightman functions are matrix elements, we employ Equation (12) to state the CPT theorem as

$$
\left\langle 0\left|\phi\left(x_{0}\right) \phi\left(x_{1}\right) \ldots \phi\left(x_{n}\right)\right| 0\right\rangle=\left\langle 0\left|\phi^{\dagger}\left(-x_{0}\right) \phi^{\dagger}\left(-x_{1}\right) \ldots \phi^{\dagger}\left(-x_{n}\right)\right| 0\right\rangle^{*},
$$

where we have used Equation (5). The usual Hilbert-space properties give $\left\langle 0\left|\phi^{\dagger}\left(-x_{0}\right) \ldots \phi^{\dagger}\left(-x_{n}\right)\right| 0\right\rangle^{*}$ $=\left\langle 0\left|\left[\phi\left(-x_{n}\right) \ldots \phi\left(-x_{0}\right)\right]^{\dagger}\right| 0\right\rangle^{*}=\left\langle 0\left|\phi\left(-x_{n}\right) \ldots \phi\left(-x_{0}\right)\right| 0\right\rangle=\mathcal{W}\left(\Delta x_{n}, \ldots, \Delta x_{1}\right)$. In other words, one must show that

$$
\mathcal{W}\left(\Delta x_{1}, \Delta x_{2}, \ldots, \Delta x_{n}\right)=\mathcal{W}\left(\Delta x_{n}, \Delta x_{n-1}, \ldots, \Delta x_{1}\right)
$$

is satisfied by all Wightman functions in order to prove CPT symmetry.

Physical Lorentz symmetry. Axioms (1) and (3) imply invariance of the Wightman function under the usual physical Lorentz transformation $\Lambda$, so we can write:

$$
\mathcal{W}\left(\Delta x_{1}, \Delta x_{2}, \ldots, \Delta x_{n}\right)=\mathcal{W}\left(\Lambda \Delta x_{1}, \Lambda \Delta x_{2}, \ldots, \Lambda \Delta x_{n}\right) \quad \text { for real } x_{k} .
$$

We cannot immediately extend this equation to complex Lorentz transformations, such as the inversion in Equation (17) because $\mathcal{W}$ may have singularities, branch points, discontinuities, etc.

Energy positivity. The next idea is to resolve $\mathcal{W}$ into its Fourier components. These will contain plane-wave 4-momenta, for which we can use Axiom (4). As an example, let us sketch this idea for the particular Wightman function $\mathcal{W}(y-x)=\langle 0|\phi(x) \phi(y)| 0\rangle$ involving two field operators. Inserting a complete set of momentum eigenstates $\int d^{4} p|p\rangle\langle p|$ and employing the translation operator on both fields $\phi(x)=\exp (i P \cdot x) \phi(0) \exp (-i P \cdot x)$ and $\phi(y)=\exp (i P \cdot y) \phi(0) \exp (-i P \cdot y)$ yields

$$
\begin{aligned}
\mathcal{W}(y-x) & =\int d^{4} p\langle 0|\phi(x)| p\rangle\langle p|\phi(y)| 0\rangle \\
& =\int d^{4} p \underbrace{\langle 0| e^{i P \cdot x}}_{=\langle 0|} \phi(0) \underbrace{e^{-i P \cdot x}|p\rangle}_{=e^{-i p \cdot x}|p\rangle=\langle p| e^{i p \cdot y}}\langle\underbrace{\langle p| e^{i P \cdot y}}_{=|0\rangle} \phi(0) \underbrace{e^{-i P \cdot y}|0\rangle} \\
& =\int d^{4} p e^{i p \cdot(y-x)}|\langle p|\phi(0)| 0\rangle|^{2} .
\end{aligned}
$$

This is the desired Fourier decomposition of $\mathcal{W}(y-x)$, where we have used that $|0\rangle$ is translation invariant and that the $|p\rangle$ are momentum eigenstates $P|p\rangle=p|p\rangle$.

Since the integral remains well behaved for decaying exponentials, we may insert certain complex-valued spacetime differences $y-x \rightarrow \Delta z=\operatorname{Re}(\Delta z)+i \operatorname{Im}(\Delta z)$. Complexifying the exponent gives

$$
i p \cdot(y-x) \rightarrow i p \cdot \Delta z=i p \cdot \operatorname{Re}(\Delta z)-p \cdot \operatorname{Im}(\Delta z)=i p \cdot \operatorname{Re}(\Delta z)-\left[p^{0} \operatorname{Im}\left(\Delta z^{0}\right)-\vec{p} \cdot \operatorname{Im}(\Delta \vec{z})\right] .
$$

The only real contribution to the exponent is the square bracket term, so it must give an overall negative contribution for exponential suppression. Axiom (4) guarantees that $p$ is in the forward momentum-space lightcone, i.e., $p^{0}>0$ and $\left(p^{0}\right)^{2}>\vec{p}^{2}$. Then, the exponential decays for $\operatorname{Im}(\Delta z)$ in the forward position-space lightcone, i.e., $\operatorname{Im}\left(\Delta z^{0}\right)>0$ and $\operatorname{Im}(\Delta z)$ is timelike $\operatorname{Im}\left(\Delta z^{0}\right)^{2}>\operatorname{Im}(\Delta \vec{z})^{2}$. This reasoning can be made rigorous for all Wightman functions, so that energy positivity implies that Equation (21) remains valid for certain complex position differences:

$$
\mathcal{W}\left(\Delta z_{1}, \Delta z_{2}, \ldots, \Delta z_{n}\right)=\mathcal{W}\left(\Lambda \Delta z_{1}, \Lambda \Delta z_{2}, \ldots, \Lambda \Delta z_{n}\right) \quad \text { for } z_{k} \text { with } \operatorname{Im}\left(\Delta z_{k}\right) \text { in forward cone. }
$$

Here, the $\Lambda$ are still the usual real Lorentz transformations.

Complex Lorentz transformation. A theorem by Bargmann, Hall, and Wightman [19] now states that Equation (24) remains valid for an even larger set of complex $\Delta z$ and also for complex Lorentz transformations. This larger set consists of all the original $\Delta z_{k}$ that have their imaginary part in the 
forward cone and also all $\Delta z_{k}^{\prime}$ that can be generated from $\Delta z_{k}$ with complex Lorentz transformations $\Lambda_{c}: \Delta z_{k}^{\prime}=\Lambda_{c} \Delta z_{k}$. This set is sometimes called the "extended tube." Equation (24) therefore takes the form

$$
\mathcal{W}\left(\Delta z_{1}, \Delta z_{2}, \ldots, \Delta z_{n}\right)=\mathcal{W}\left(\Lambda_{c} \Delta z_{1}, \Lambda_{c} \Delta z_{2}, \ldots, \Lambda_{c} \Delta z_{n}\right) \quad \text { for } \Delta z_{k} \text { in extended tube. }
$$

A special case of this relation is

$$
\mathcal{W}\left(\Delta z_{1}, \Delta z_{2}, \ldots, \Delta z_{n}\right)=\mathcal{W}\left(-\Delta z_{1},-\Delta z_{2}, \ldots,-\Delta z_{n}\right) \quad \text { for } \Delta z_{k} \text { in extended tube, }
$$

where we plugged in the specially chosen complex Lorentz transformation (17), i.e., $\Lambda_{c}=-\mathbb{1}$.

Another important difference between Equations (24) and (25), which follows from the Bargmann-Hall-Wightman theorem, is that there are no real-valued, physical $\Delta z_{k}$ in Equation (24) because the imaginary parts of these $\Delta z_{k}$ are all timelike vectors and therefore strictly nonzero. However, Equation (25) holds for some real-valued spacetime points: the extended tube contains not only the above complex $\Delta z_{k}$, but also transformed points $\Lambda_{c} \Delta z_{k}$, and a complex Lorentz transformation acting on a complex $\Delta z_{k}$ can give a real four-vector. Consider the example of $\Delta z=(i, 0,0,0)$. Clearly, $\operatorname{Im} \Delta z=(1,0,0,0)$ is in the forward cone, so it is valid input for Equation (24). For Equation (25), we may transform $\Delta z$ to $\Delta y$ by any complex $\Lambda_{c}$, so, in particular, we may select an imaginary boost $w=\frac{1}{2} i \pi$

$$
\Delta y=\Lambda\left(w=\frac{1}{2} i \pi, \alpha=0\right) \Delta z=\left(\begin{array}{llll}
0 & i & 0 & 0 \\
i & 0 & 0 & 0 \\
0 & 0 & 1 & 0 \\
0 & 0 & 0 & 1
\end{array}\right)\left(\begin{array}{l}
i \\
0 \\
0 \\
0
\end{array}\right)=\left(\begin{array}{c}
0 \\
-1 \\
0 \\
0
\end{array}\right)
$$

These special, physical points $\Delta y_{k}$ in the extended tube are called Jost points. A special case of Equation (26) is therefore

$$
\mathcal{W}\left(\Delta y_{1}, \Delta y_{2}, \ldots, \Delta y_{n}\right)=\mathcal{W}\left(-\Delta y_{1},-\Delta y_{2}, \ldots,-\Delta y_{n}\right) \quad \text { at Jost points. }
$$

By using matrix manipulations, Jost showed that all $\Delta y_{k}$ are not only real but also spacelike. The significance of this result is that it permits the application of Axiom (5).

Microscopic causality and spin-statistics. Translating Equation (28) back to vacuum expectation values gives

$$
\left\langle 0\left|\phi\left(y_{0}\right) \phi\left(y_{1}\right) \ldots \phi\left(y_{n}\right)\right| 0\right\rangle=\left\langle 0\left|\phi\left(-y_{0}\right) \phi\left(-y_{1}\right) \ldots \phi\left(-y_{n}\right)\right| 0\right\rangle \quad \text { at Jost points, }
$$

where $\Delta y_{k}=y_{k}-y_{k-1}$. The CPT theorem (19) contains the Hermitian-conjugate fields, so we can use the general Hilbert-space scalar-product property $\langle\psi|\mathcal{A}| \chi\rangle=\left\langle\chi\left|\mathcal{A}^{\dagger}\right| \psi\right\rangle^{*}$ on the right-hand side of Equation (29) to write

$$
\left\langle 0\left|\phi\left(y_{0}\right) \phi\left(y_{1}\right) \ldots \phi\left(y_{n}\right)\right| 0\right\rangle=\left\langle 0\left|\phi^{\dagger}\left(-y_{n}\right) \phi^{\dagger}\left(-y_{n-1}\right) \ldots \phi^{\dagger}\left(-y_{0}\right)\right| 0\right\rangle^{*} \quad \text { at Jost points. }
$$

However, this leaves the fields on the right-hand side in the reverse order relative to Equation (19) that we want to prove. However, since the Jost points are real and mutually spacelike separated, we can use the commutativity property of Axiom (5) to change the order of the fields freely:

$$
\left\langle 0\left|\phi\left(y_{0}\right) \phi\left(y_{1}\right) \ldots \phi\left(y_{n}\right)\right| 0\right\rangle=\left\langle 0\left|\phi^{\dagger}\left(-y_{0}\right) \phi^{\dagger}\left(-y_{1}\right) \ldots \phi^{\dagger}\left(-y_{n}\right)\right| 0\right\rangle^{*} \quad \text { at Jost points. }
$$

As before, we can express this in terms of Wightman functions using $\left\langle 0\left|\phi^{\dagger}\left(-y_{0}\right) \ldots \phi^{\dagger}\left(-y_{n}\right)\right| 0\right\rangle^{*}$ $=\left\langle 0\left|\left[\phi\left(-y_{n}\right) \ldots \phi\left(-y_{0}\right)\right]^{\dagger}\right| 0\right\rangle^{*}=\left\langle 0\left|\phi\left(-y_{n}\right) \ldots \phi\left(-y_{0}\right)\right| 0\right\rangle=\mathcal{W}\left(\Delta y_{n}, \ldots, \Delta y_{1}\right)$ :

$$
\mathcal{W}\left(\Delta y_{1}, \Delta y_{2}, \ldots, \Delta y_{n}\right)=\mathcal{W}\left(\Delta y_{n}, \Delta y_{n-1}, \ldots, \Delta y_{1}\right) \quad \text { at Jost points. }
$$


We remark that this reordering implicitly uses the spin-statistics theorem, which can be proved independently. If we had also considered half-integer spin fields, anticommutators would generate a factor of -1 for each interchange of two fermionic fields.

Each of the two Wightman functions in Equation (32) have their respective analytic continuation into the extended tube by the above line of reasoning. However, these analytic continuations need not be the same a priori because the two functions only agree at Jost points, and not for all real $\Delta x_{k}$. However, it can be shown that the Jost points form an open set, and there is a mathematical theorem that guarantees the equality of analytic functions if they agree in a real-valued open set. Thus, the two analytic continuations are, in fact, identical

$$
\mathcal{W}\left(\Delta z_{1}, \Delta z_{2}, \ldots, \Delta z_{n}\right)=\mathcal{W}\left(\Delta z_{n}, \Delta z_{n-1}, \ldots, \Delta z_{1}\right) \quad \text { for } \Delta z_{k} \text { in extended tube. }
$$

One can now take the limit of physical spacetime positions $\Delta z_{k} \rightarrow \Delta x_{k}$ :

$$
\mathcal{W}\left(\Delta x_{1}, \Delta x_{2}, \ldots, \Delta x_{n}\right)=\mathcal{W}\left(\Delta x_{n}, \Delta x_{n-1}, \ldots, \Delta x_{1}\right) \quad \text { for real } \Delta x_{k} \text {. }
$$

This is the result we needed to prove. Although more technical than the Lagrangian proof, the above line of reasoning in axiomatic field theory reduces the requirements for CPT symmetry down to the bare essentials, namely quantum physics, Lorentz symmetry, energy positivity, and microcausality.

\section{Some Physical Consequences of CPT Symmetry}

The Standard Model of particle physics, and indeed many theoretical approaches to unify nongravitational interactions, satisfy the ingredients of the CPT theorem and are therefore CPT invariant. It is then natural to inquire about the physical consequences of CPT symmetry. Since the CPT transformation contains charge conjugation linking particles to antiparticles, CPT invariance is expected to provide a correlation between the properties of matter and antimatter. In what follows, we briefly recall key predictions of CPT symmetry.

Any physics prediction typically requires some form of theoretical framework. In what follows, we will often rely on general features of a relativistic quantum theory. For example, we assume that a single Hamiltonian operator $H=P^{0}$ governs the dynamics of both particles and antiparticles. For such a system, it is meaningful to predict properties of antimatter from those of matter using the CPT symmetry of $H$. Examples include the quantum-mechanical Dirac Hamiltonian as well as Hamiltonians constructed in quantum field theory. On the other hand, the usual non-relativistic single-particle Hamiltonians present in the Schrödinger or the Pauli equation are unsuitable in the present context: The corresponding antiparticle Hamiltonians must essentially be specified independently, so that antiparticle properties cannot convincingly be considered predictions of the theory. With this in mind, our goal is to ask the following:

$$
\begin{aligned}
& \text { Given } C P T \text { symmetry } H=\Theta H \Theta^{\dagger} \text {, how are the } \\
& \text { properties of matter and antimatter states related? }
\end{aligned}
$$

There are various ways in which matter and antimatter states can be compared. Here, we discuss three of the most common ones. They concern properties of stationary states, observables related to dynamical processes resulting from the time evolution of non-stationary states, and coupling constants. We will see that the general line of inquiry (35) needs to be modified for couplings indicating a qualitative difference with respect to the first two types of CPT-symmetry implications.

\subsection{CPT-Symmetry Implications for Eigenstates}

A particularly interesting set of quantum states are eigenstates of $H$. These are usually labeled by quantum numbers such as charge, momentum, spin, intrinsic parity, isospin, etc. Thus, a first context for question (35) is provided by the implications of CPT symmetry for the relation between particle and 
antiparticle quantum numbers. In what follows, we will focus on quantum numbers that arise from exact continuous symmetries. By Noether's theorem, these symmetries are associated with conserved current densities, and in the absence of spontaneous symmetry breaking, these current densities can be used to construct the spacetime-independent Hermitian operators that give rise to the quantum numbers in question. We are thus led to investigate the CPT properties of these conserved quantities.

In addition to the presumed translation and Lorentz invariance, internal symmetries, such as gauge symmetries, occur often in physics. The current densities are basically local products of fields and field derivatives, so their behavior under CPT is determined by Equation (9). In particular, we do not need their explicit, model-dependent form, but only their Lorentz-index structure. This structure is taken to be determined solely by the indices on dynamical fields and four-gradients; nondymanical objects with Lorentz indices, such as background fields, are excluded in the following:

$$
\begin{array}{lll}
\text { internal: } & \text { current } j^{\mu}(x) & \Theta j^{\mu}(x) \Theta^{+}=-j^{\mu}(-x), \\
\text { translations: } & \text { energy-momentum tensor } T^{\mu v}(x) & \Theta T^{\mu \nu}(x) \Theta^{+}=T^{\mu \nu}(-x), \\
\text { rotations and boosts: } & \text { angular-momentum tensor } \mathcal{J}^{\mu \nu \rho}(x) & \Theta \mathcal{J}^{\mu \nu \rho}(x) \Theta^{+}=-\mathcal{J}^{\mu \nu \rho}(-x) .
\end{array}
$$

Here, we have used that all three current densities are Hermitian, as expected for physical observables. The internal-symmetry current density $j^{\mu}$ is not necessarily the usual electromagnetic current. It could also be associated with other internal symmetries. In fact, if there are $n$ independent unbroken internal symmetries, there are $n$ independent conserved currents $j_{n}^{\mu}(x)$ [20].

Standard arguments now yield the associated conserved operators as spatial integrals of the currents. For example,

$$
\begin{aligned}
Q & =\int d^{3} x j^{0}(t, \vec{x}), \\
P^{\mu} & =\int d^{3} x T^{0 \mu}(t, \vec{x}), \\
M^{\mu v} & =\int d^{3} x \mathcal{J}^{0 \mu v}(t, \vec{x}),
\end{aligned}
$$

where we have chosen to arrange the expressions for the current densities such that they are conserved in their first index. We remark in passing that the tensor $M^{\mu v}$ is antisymmetric, so it has six independent components resulting from three independent boosts and rotations. The three purely spatial components represent the usual total angular momentum $\vec{J}$, with $J^{k}=\frac{1}{2} \epsilon^{k l m} M^{l m}$.

The CPT transformations (36) together with the expressions (37) determine the behavior of charges, momenta, and angular momenta under CPT. For example,

$$
\begin{aligned}
\Theta Q \Theta^{+} & =-\int d^{3} x j^{0}(-t,-\vec{x}) \\
& =-\int_{-\infty}^{+\infty} \int_{-\infty}^{+\infty} \int_{-\infty}^{+\infty} d x^{1} d x^{2} d x^{3} j^{0}(-t,-\vec{x}) \\
& =\int_{+\infty}^{-\infty} \int_{+\infty}^{-\infty} \int_{+\infty}^{-\infty} d y^{1} d y^{2} d y^{3} j^{0}(-t, \vec{y}) \\
& =-\int_{-\infty}^{+\infty} \int_{-\infty}^{+\infty} \int_{-\infty}^{+\infty} d y^{1} d y^{2} d y^{3} j^{0}(-t, \vec{y}) \\
& =-Q,
\end{aligned}
$$


where we have adopted Cartesian coordinates, changed the integration variable $\vec{x} \rightarrow-\vec{y}$, and used the time independence of $Q$. Analogously, one can show

$$
\begin{aligned}
\Theta P^{\mu} \Theta^{\dagger} & =P^{\mu}, \\
\Theta M^{\mu v} \Theta^{\dagger} & =-M^{\mu v} .
\end{aligned}
$$

In particular, this implies that the angular-momentum operator $\vec{J}$ changes sign under CPT.

We continue by considering an arbitrary asymptotic one-particle state $\left|p^{\mu}, j(j+1), j_{p}, q\right\rangle$ labeled by its four-momentum $p^{\mu}$, its angular-momentum square $j(j+1)$, its angular-momentum projection $j_{p}$ along $\vec{p}$, and its charge $q$. The corresponding antiparticle state has then the following quantum numbers:

$$
\Theta\left|p^{\mu}, j, j_{p}, q\right\rangle=\left|p^{\mu}, j,-j_{p},-q\right\rangle .
$$

This equation follows from the transformation behavior (38)-(40) of the corresponding operators. We demonstrate this for the case of $p^{\mu}$; the other quantum numbers can be treated analogously. The goal is the determination of the four-momentum eigenvalue of the antiparticle state $\Theta\left|p^{\mu}, \ldots\right\rangle$ given that the particle state satisfies $P^{\mu}\left|p^{\mu}, \ldots\right\rangle=p^{\mu}\left|p^{\mu}, \ldots\right\rangle$. We have:

$$
\begin{aligned}
P^{\mu}\left|p^{\mu}, \ldots\right\rangle & =p^{\mu}\left|p^{\mu}, \ldots\right\rangle, \quad \text { so that } \\
\underbrace{\Theta P^{\mu} \Theta^{\dagger}}_{P^{\mu}} \Theta\left|p^{\mu}, \ldots\right\rangle & =p^{\mu} \Theta\left|p^{\mu}, \ldots\right\rangle, \\
P^{\mu} \Theta\left|p^{\mu}, \ldots\right\rangle & =p^{\mu} \Theta\left|p^{\mu}, \ldots\right\rangle .
\end{aligned}
$$

The crucial step here is using the CPT transformation of the momentum operator $\Theta P^{\mu} \Theta^{\dagger}=P^{\mu}$; it follows from Equation (39).

The CPT transformations (41) of the one-particle quantum numbers involve the total angular momentum $\vec{J}=\vec{L}+\vec{S}$, which is composed of the orbital angular momentum $\vec{L}$ and the state's intrinsic spin $\vec{S}$. The transformation of the more customary spin quantum numbers can be made plausible using classical-physics arguments: $\dot{\vec{L}}=\dot{\vec{r}} \times \vec{p}=\dot{\vec{r}} \times(m \dot{\vec{r}})=\overrightarrow{0}$, where we have used that $\vec{p}$ is constant in time. Note also that the projection of $\vec{J}$ onto $\vec{p}$ is independent of $\vec{L}$ since $\vec{L} \cdot \vec{p}=(\vec{r} \times \vec{p}) \cdot \vec{p}=0$. This supports the fact that spin is separately conserved for a free particle, and its quantum numbers can therefore be used to label single-particle states. To determine the behavior of spin under CPT, note that the corresponding spin contribution to the angular-momentum tensor $\mathcal{J}^{\mu v \rho}$ will also have three Lorentz indices. Hence, it will exhibit the same CPT behavior as $\mathcal{J}^{\mu v \rho}$, so that Equation (40) continues to hold for the particle's spin by itself. We can therefore recast Equation (41) as

$$
\Theta\left|p^{\mu}, s_{,} s_{p}, q\right\rangle=\left|p^{\mu}, s_{,}-s_{p},-q\right\rangle \text {. }
$$

The spin projection $s_{p}$ onto the momentum $\vec{p}$ can be identified with the state's helicity. The above reasoning for the spin properties under $\mathrm{CPT}$ can be established more rigorously using the Pauli-Lubanski vector $W_{\mu}=\frac{1}{2} \epsilon_{\mu v \rho \sigma} M^{v \rho} P^{\sigma}$ [8].

We mention in passing two immediate consequences of the above results. First, assuming that Lorentz symmetry holds and we can identify $\sqrt{p^{\mu} p_{\mu}}=m$ or $p^{0}(\vec{p}=\overrightarrow{0})=E(\vec{p}=\overrightarrow{0})=m$ with the particles' invariant masses, it is apparent that particle and antiparticle states must have the same mass [21]. Second, the reasoning (42) is general enough to hold also for multiparticle states. In particular, consider the zeroth-component of (42). Since $P^{0}=H$ is the Hamiltonian and $p^{0}=E$ an energy eigenvalue, one recognizes the usual stationary Schrödinger-type energy-eigenvalue equation. We may therefore conclude that if there is a matter bound state $|n\rangle$ with eigenvalue $E_{n}$, there is also an antimatter bound state $|\bar{n}\rangle=\Theta|n\rangle$ with the same eigenvalue $E_{n}$. Thus, any matter bound state will possess the same energy spectrum as the corresponding antimatter bound state. 


\subsection{CPT-Symmetry Implications for the Dynamics of Nonstationary States}

Next, consider quantum states with a nontrivial time evolution. In this context, question (35) leads us to compare dynamical properties of matter and antimatter states, such as transition rates, cross sections, branching ratios, etc. As an example, we consider the lifetime $\tau$ of an unstable particle. A leading-order expression for its decay rate may often be obtained from Fermi's Golden Rule:

$$
\Gamma_{i \rightarrow f}=\tau^{-1}=2 \pi\left|\left\langle f\left|H_{\text {int }}\right| i\right\rangle\right|^{2} \rho\left(E_{f}\right),
$$

where $i$ and $f$ refer to initial and final configurations, respectively, with $|i\rangle$ and $|f\rangle$ eigenstates of some CPT-invariant, free Hamiltonian $H$. The decay proceeds through an interaction Hamiltonian $H_{\text {int }}$ presumed to exhibit no explicit time dependence. In the Schrödinger picture, which is the required form for Equation (44), $H_{\text {int }}$ is therefore constant in time. The density of final states $\rho(E)$ is defined as the number of states $d n=\rho(E) d E$ in the energy interval $d E$; it essentially provides a measure of the phase space accessible to the decay products. Analogously, the antiparticle decay rate is

$$
\Gamma_{\bar{\imath} \rightarrow \bar{f}}=\bar{\tau}^{-1}=2 \pi\left|\left\langle\bar{f}\left|H_{\text {int }}\right| \bar{i}\right\rangle\right|^{2} \bar{\rho}\left(E_{\bar{f}}\right),
$$

where $\bar{\imath}$ and $\bar{f}$ denote the corresponding initial and final antiparticle configurations, $\bar{\tau}$ the antiparticle lifetime, and $\bar{\rho}\left(E_{\bar{f}}\right)$ density of final antiparticle states. According to our above analysis, the kinematics of the antiparticle's decay process is essentially identical to that of the particle [22], so that we may conclude that the final-state energies as well as the density of final states remain unchanged $\bar{\rho}\left(E_{\bar{f}}\right)=\rho\left(E_{f}\right)$. With $|\bar{\imath}\rangle=|\Theta i\rangle,|\bar{f}\rangle=|\Theta f\rangle$, and Equation (12), we have

$$
\bar{\tau}^{-1}=2 \pi\left|\left\langle f\left|\Theta^{\dagger} H_{\text {int }} \Theta\right| i\right\rangle^{*}\right|^{2} \rho\left(E_{f}\right)=2 \pi\left|\left\langle f\left|H_{\text {int }}\right| i\right\rangle\right|^{2} \rho\left(E_{f}\right)=\tau^{-1},
$$

where we have used CPT invariance $\Theta^{\dagger} H_{\text {int }} \Theta=H_{\text {int }}$ in the second step. Thus, the lifetimes of particle and antiparticle agree, at least at leading order in perturbation theory.

The above arguments for the CPT-transformation behavior of the various quantum numbers and the decay time involve to some extent Lagrangian field theory and the canonical formalism, and they sidestep intricate issues regarding the theoretical treatment of unstable particles in quantum field theory. We mention in passing that these arguments can be made more rigorous [23]. For example, the momentum-space propagator for free particles contains the factor $S_{\text {free }}\left(p^{2}\right) \sim\left(p^{\mu} p_{\mu}-m^{2}\right)^{-1}$, so that $S_{\text {free }}$ has a pole at the particle's squared mass. The propagator $S_{\text {full }}$ of a fully interacting theory will then in general have a pole at some complex $p^{2}=\left(m-\frac{1}{2} i \Gamma\right)^{2}$ [24]. As the notation suggests, the imaginary part of such a pole is tied to the decay rate of the particle: $\mathrm{A} \vec{p}=\overrightarrow{0}$ plane-wave solution will have $\omega=E=\left(m-\frac{1}{2} i \Gamma\right)$, leading to an exponentially decaying solution $\exp (-i \omega t)=$ $\exp \left(-\frac{1}{2} \Gamma t\right) \exp (-i m t)$. The probability for the particle state $\exp \left(-\frac{1}{2} \Gamma t\right) \exp (-i m t)|i\rangle$ after time $t$ to remain in the initial state $|i\rangle$ is then $\left|\left\langle i\left|\exp \left(-\frac{1}{2} \Gamma t\right) \exp (-i m t)\right| i\right\rangle\right|^{2}=\exp (-\Gamma t)$, so that $\Gamma=\tau^{-1}$ can be interpreted as the decay rate. More careful arguments show that the measured mass and lifetime are indeed determined by the real and imaginary parts of the pole, respectively [25]. Since the propagator is a specific combination of momentum-space Wightman functions, the CPT transformation of mass and lifetime can be studied nonperturbatively without assuming a Lagrangian density with the same results $m=\bar{m}$ and $\tau=\bar{\tau}[26]$.

\subsection{CPT-Symmetry Implications for Couplings}

The relation between a given coupling constant pertaining to matter and the corresponding one for antimatter is often considered to be a third type of consequence following from CPT symmetry. To gain further insight, some preliminary considerations are necessary. One of these concerns the definition of what actually constitutes a coupling constant. Typically, a coupling constant is thought of as a number that parametrizes in one form or another the strength of a specific type 
of interaction. Numerical factors-possibly dimensionful-multiplying expressions of dynamical variables in a Hamiltonian are prime candidates for being interpreted as coupling constants.

The above definition clearly needs further refinements. As one such refinement, we exclude pure normalization factors. For example, the usual factor of one half in $\frac{1}{2} \frac{p^{2}}{m}$ in a nonrelativistic Hamiltonian normalizes the kinetic energy relative to the potential energy and is therefore not usually considered to be a coupling.

A second issue concerns the ambiguity in splitting an interaction term into a coupling factor $a$ and an operator factor $\mathcal{A}$ : selecting an arbitrary number $b$ and defining a new coupling $a^{\prime} \equiv b a$ and a new operator $\mathcal{A}^{\prime} \equiv b^{-1} \mathcal{A}$, it is clear that $a^{\prime} \mathcal{A}^{\prime}$ represents the same overall Hamiltonian contribution. Note that implies one can always choose $a$ to be real valued. Such a choice is natural in the sense that if the interaction $a \mathcal{A}$ is Hermitian and $a$ is real, then the operator $\mathcal{A}$ is Hermitian.

A third question involves the specification of what an interaction term is. The conventional decomposition of quantum-field Lagrangians into free-field and interaction terms is based partly on considerations that are practical for perturbation theory. A bare-mass parameter $m_{b}$, for instance, (which is, in general, different from the actual particle mass $m$ ) would then be part of the free-field terms. However, in many circumstances the corresponding term can equivalently be treated as an interaction in the sense of perturbation theory with $m_{b}$ measuring the strength of this self interaction.

As a fourth question, one might want to consider whether the theory permits the free choice of a given interaction strength. For example, the structure of the Standard Model dictates a relationship between the electromagnetic couplings of charged leptons and quarks.

Another fifth issue to address is the meaning of matter coupling constant versus antimatter coupling constant. For a particle in an external field, there is an intuitive notion for this concept: the particle and antiparticle could have the same type of interaction with the external field (e.g., they both couple linearly via their spin), but the strength of their respective couplings may differ. However, in a field-theory Lagrangian, for example, the product of $n$ fields can often be interpreted as a simultaneous interaction of multiple particles and antiparticles whose numbers add up to $n$. In such a scenario, the notion of pure matter versus pure antimatter coupling is blurred at best.

A sixth important aspect worth mentioning here concerns couplings specifically in the framework of quantum field theory. In this framework, coupling parameters can often be interpreted to depend on the energy scale at which the parameter is measured. Although this effect arises through renormalization theory and is thus quite well understood, it does add an additional level of interpretational complexity.

These remarks illustrate that a comprehensive study of the consequences of CPT symmetry for couplings would need to be preceded by in-depth discussions of a number of preparatory issues. Addressing these in detail lies outside the scope of the present work. However, for definiteness, let us consider all physical spacetime-constant model parameters of a theory that have been made real valued and unambiguous by suitable prescriptions, which cannot be fixed by theoretical considerations alone, and need to be measured (and actually can be measured) as coupling constants.

Regardless of the details of the definition of coupling constants, the above discussion highlights two important points. First, extending the study of the consequences of CPT symmetry to couplings necessarily introduces an additional layer of theory dependence. This contrasts our previous results, such as Equations (41), (43), and (46) for stationary and time-dependent states. These relied only on a few basic physical assumptions, such as the validity of quantum theory, Lorentz and translation invariance, and internal symmetries. However, there are an infinite number of interactions compatible with these symmetries, and each of these interactions comes with their own respective coupling strength subject to suitable definitions and refinements, such as those discussed above. Second, the previously adopted line of investigation (35) becomes less meaningful since couplings are input parameters rather than predictions of the model; quantum states are not usually labeled by coupling constants [27]; and the reasoning $\Theta b \Theta^{\dagger}=b$ for real-valued couplings is meaningless. 
One possible way to proceed is to modify the original approach (35) and ask if a CPT symmetric Hamiltonian necessarily needs to have a single coupling strength for a given interaction for both matter and antimatter. It would then seem desirable to obtain further insight into the above fifth issue and develop a theoretical notion to distinguish between matter and antimatter couplings present in a single Hamiltonian describing both types of matter. In a conventional field-theory Lagrangian, the matter and antimatter physics is contained in a single quantum field. An interaction is conventionally modeled by a given product of these fields. Thus, this single product term parametrized by a single coupling describes the interaction of both particles and antiparticles. In this sense, the equality of matter and antimatter couplings becomes almost trivial.

A somewhat more general reasoning for the equality of couplings may be obtained as follows. Consider the Hamiltonian [28]

$$
H(\vec{x})=H_{0}(x)+a \mathcal{A}(x)+\bar{a} \overline{\mathcal{A}}(x) .
$$

Here, $\mathcal{A}(x)$ may depend on the spacetime point $x^{\mu}=(t, \vec{x})$ and represents the operator for a given type of interaction, such as a spin coupled to the magnetic field, and $\overline{\mathcal{A}}(x) \equiv \mathcal{A}(x)_{\mathrm{CPT}}=\Theta \mathcal{A}(x) \Theta^{\dagger}$ is its CPT conjugate. Note that the special case $\mathcal{A}(x)-\mathcal{A}(x)=0$ is not assumed. The corresponding coupling constants $a$ and $\bar{a}$ are independent and may differ. The remaining part of the Hamiltonian is denoted by $H_{0}(x)$ and could, for example, include the free-particle physics and other interactions. We further take the theory to be time-translation invariant, so that $H$ is conserved and generates time translations via the unitary operator $\exp (-i H t)$.

Before we continue and study the implications for $a$ and $\bar{a}$ when CPT symmetry is imposed on $H$, we pause here to gain confidence that the interaction term $H_{\text {int }}(x)=a \mathcal{A}(x)+\bar{a} \overline{\mathcal{A}}(x)$ indeed treats matter and antimatter differently. In particular, we seek to verify that the intuitive notion for the single-particle case alluded to in the fifth issue above, is described by $H_{\text {int }}$ as a special limit. In other words, we want to show that single particles and single antiparticles obey the same type of interaction, but with a different coupling strengths. To see this, consider the limit of one-particle states, and in particular the situation in which the general structure of the operator $\mathcal{A}$ does not contribute to the antiparticle interactions, so that $\left\langle\bar{n}|\mathcal{A}| \bar{n}^{\prime}\right\rangle=0$ for any pair of single-antiparticle states $|\bar{n}\rangle$ and $\left|\bar{n}^{\prime}\right\rangle$. Conversely, $\overline{\mathcal{A}}$ is then irrelevant for particle interactions: $0=\left\langle\bar{n}|\mathcal{A}| \bar{n}^{\prime}\right\rangle=\left\langle\Theta n|\mathcal{A}| \Theta n^{\prime}\right\rangle=$ $\left\langle n\left|\Theta^{\dagger} \mathcal{A} \Theta\right| n^{\prime}\right\rangle^{*}=\left\langle n|\overline{\mathcal{A}}| n^{\prime}\right\rangle^{*}$ for any two particle states $|n\rangle$ and $\left|n^{\prime}\right\rangle$. The particle's interactions are now completely specified by the matrix elements $\left\langle n\left|H_{\text {int }}\right| n^{\prime}\right\rangle=a\left\langle n|\mathcal{A}| n^{\prime}\right\rangle=a A_{n n^{\prime}}$, and the CPT-conjugate situation for antiparticles is governed by $\left\langle\bar{n}^{\prime}\left|H_{\text {int }}\right| \bar{n}\right\rangle=\bar{a}\left\langle\bar{n}^{\prime}|\overline{\mathcal{A}}| \bar{n}\right\rangle=\bar{a}\left\langle\bar{n}^{\prime}\left|\Theta^{\dagger} \mathcal{A} \Theta\right| \bar{n}\right\rangle=$ $\bar{a}\left\langle\Theta \bar{n}^{\prime}|\mathcal{A}| \Theta \bar{n}\right\rangle^{*}=\bar{a}\left\langle n^{\prime}|\mathcal{A}| n\right\rangle^{*}=\bar{a}\left\langle n\left|\mathcal{A}^{\dagger}\right| n^{\prime}\right\rangle=\bar{a} A_{n n^{\prime}}$, where the hermiticity of $\mathcal{A}$ has been used in the last step, and where we have allowed for time-dependent effects by reversing initial and final states. This demonstrates that both particles and antiparticles have the same type of interaction represented by $A_{n n^{\prime}}$, but their respective couplings $a$ and $\bar{a}$ are independent. This reasoning can be extended to multiparticle states containing both matter and antimatter and more general $\mathcal{A}$ : the corresponding CPT conjugate state will have different interaction matrix elements if $a \neq \bar{a}$.

We may now proceed with confidence that the interaction (47) indeed represents a general theoretical description for distinguishing between matter and antimatter couplings. More specifically, we can now study in a meaningful way the relation between $a$ and $\bar{a}$ imposed by CPT symmetry. Clearly, in the special case $\mathcal{A}(x)=\overline{\mathcal{A}}(x)$, we have $H_{\text {int }}(x)=(a+\bar{a}) \mathcal{A}(x)$, which corresponds to at most one measurable coupling $a^{\prime}=(a+\bar{a})$, and there is nothing to prove. In what follows, we therefore focus on the nontrivial case, in which $\mathcal{A}\left(x_{0}\right)-\overline{\mathcal{A}}\left(x_{0}\right) \neq 0$ at least for some $x=x_{0}$. The existence of a unitary time-translation operator then guarantees that

$$
\mathcal{A}\left(0, \vec{x}_{0}\right)-\overline{\mathcal{A}}\left(0, \vec{x}_{0}\right) \neq 0 .
$$

This follows because any unitary transformation of a nonzero operator must give another nonzero operator. 
CPT symmetry requires the CPT conjugate Hamiltonian

$$
\bar{H}(\vec{x})=\bar{H}_{0}(x)+a \overline{\mathcal{A}}(t)+\bar{a} \mathcal{A}(t)
$$

to be equal to $H(\vec{x})$. Here, we have denoted $\bar{H}_{0}(x) \equiv \Theta H_{0}(x) \Theta^{\dagger}$. To implement CPT symmetry, we equate the right-hand sides of Equations (47) and (49):

$$
H_{0}(x)+a \mathcal{A}(x)+\bar{a} \overline{\mathcal{A}}(x)=\bar{H}_{0}(x)+a \overline{\mathcal{A}}(x)+\bar{a} \mathcal{A}(x) .
$$

To relate $H_{0}(x)$ and $\bar{H}_{0}(x)$, it is useful to display their time dependences explicitly using the time-translation operator:

$$
e^{i H t} H_{0}(0, \vec{x}) e^{-i H t}+a \mathcal{A}(x)+\bar{a} \overline{\mathcal{A}}(x)=e^{-i H t} \bar{H}_{0}(0, \vec{x}) e^{i H t}+a \overline{\mathcal{A}}(x)+\bar{a} \mathcal{A}(x) .
$$

Note that according to its definition, the time evolution of $\bar{H}_{0}(t, \vec{x})$ must be determined by CPT-conjugating the time evolution of $H_{0}(t, \vec{x})$. Subsequently, one then again use CPT invariance $H=\bar{H}$ to arrive at Equation (51).

With our definition of coupling constant, $a$ and $\bar{a}$ are free model parameters not determined within the theory. We can therefore expect a self-consistent theory for large ranges of $a$ and $\bar{a}$. In particular, Equation (51) should remain valid in the special case of zero-strength interactions $a=\bar{a}=0$ :

$$
e^{i H t} H_{0}(0, \vec{x}) e^{-i H t}=e^{-i H t} \bar{H}_{0}(0, \vec{x}) e^{i H t} .
$$

While this limit affects the expression for $H$, and thus the time-evolution operator [29], both $H_{0}(0, \vec{x})$ and $\bar{H}_{0}(0, \vec{x})$ are left unchanged: $H_{0}(0, \vec{x})$ and $\bar{H}_{0}(0, \vec{x})$ can be interpreted a Schrödinger-picture operators, so that the time evolution of matrix elements is entirely contained in the states. In particular, $H_{0}(0, \vec{x})$ and $\bar{H}_{0}(0, \vec{x})$ are free of any dependence on other parts of $H$, such as $a$ and $\bar{a}$. Setting $t=0$, we find that $H_{0}(0, \vec{x})=\bar{H}_{0}(0, \vec{x})$. Since both of these are constant in time, we may conclude that

$$
H_{0}(0, \vec{x})=\bar{H}_{0}(0, \vec{x})
$$

holds in general for all $t, a$, and $\bar{a}$. Equation (51) then becomes

$$
e^{i H t} H_{0}(0, \vec{x}) e^{-i H t}+a \mathcal{A}(x)+\bar{a} \overline{\mathcal{A}}(x)=e^{-i H t} H_{0}(0, \vec{x}) e^{i H t}+a \overline{\mathcal{A}}(x)+\bar{a} \mathcal{A}(x) .
$$

At $t=0$ and $\vec{x}=\vec{x}_{0}$ the $H_{0}$ terms cancel, and we find $a \mathcal{A}\left(0, \vec{x}_{0}\right)+\bar{a} \overline{\mathcal{A}}\left(0, \vec{x}_{0}\right)=a \overline{\mathcal{A}}\left(0, \vec{x}_{0}\right)+\bar{a} \mathcal{A}\left(0, \vec{x}_{0}\right)$, or

$$
a\left[\mathcal{A}\left(0, \vec{x}_{0}\right)-\overline{\mathcal{A}}\left(0, \vec{x}_{0}\right)\right]=\bar{a}\left[\mathcal{A}\left(0, \vec{x}_{0}\right)-\overline{\mathcal{A}}\left(0, \vec{x}_{0}\right)\right] .
$$

Recalling the condition (48), Equation (55) is therefore only satisfied if

$$
a=\bar{a},
$$

i.e., matter and antimatter couplings must be equal. We stress that the reasoning leading to this conclusion may not be the most general one. Nevertheless, a number of additional considerations relative to the properties of quantum numbers and particle lifetimes under CPT seem to be necessary.

Another perspective on the equality of coupling constants for matter and antimatter is provided by the following reasoning. From a more experimental viewpoint, the measurement of a coupling typically involves the properties of eigenstates or time-dependent states, such as spectra of confined particles or cross sections, respectively. Carefully chosen systems and measurements then permit the extraction of the value of a particular coupling. For example, the energy levels of a charged particle in a Penning trap yield the $g$ factor of the particle, and from the decay cross section of the muon (i.e., the muon lifetime) the Fermi constant can be determined. We had earlier argued that CPT invariance guarantees 
the equality of spectra and decay times for a system and its CPT-conjugate counterpart. We remind the reader that the equality between these primary experimental observables depends only on general physical features, such as the validity of quantum physics, Lorentz and translation invariance, and internal symmetries [30]. In particular, this equality is independent of the nature and the number of the interactions relevant in the given experimental situation. If, in addition, a theoretical prediction for the spectrum or the cross section in terms of specific interactions is known, the corresponding coupling can be extracted. Assuming CPT invariance, the extraction procedure for a given physical system and its CPT conjugate would, in essence, have to agree. This agreement together with the equality of spectra and cross sections leads to identical couplings for matter and antimatter. Note, however, that this idea requires additional theoretical input linking the interactions to the physical observables. For example, in the Penning-trap case mentioned above, it must be postulated that the magnetic-field interaction of the particle is of the specific form $g \mu \vec{s} \cdot \vec{B}$ to determine $g$. This reiterates the above point that the equality of coupling constants represents a more theory-dependent result than the equality of spectra, lifetimes, etc.

\section{CPT Violation}

The proof of the CPT theorem highlights the fundamental status of CPT symmetry. Tests of such basic physics laws are interesting because they may either solidify the foundations of physics further, or they may uncover fundamentally new physics. From a theoretical perspective, one might then ask if and how CPT symmetry can be evaded in a physically acceptable manner. The proof of the CPT theorem suggests three broad overlapping approaches to this question.

The first of these approaches is based on the fact that CPT holds in the framework of point-particle quantum field theory. Thus, CPT symmetry could be avoided if more fundamental physics requires a broader framework that contains quantum field theory only as a limiting case. One example in this context is string theory. Indeed, it is known that in the field theory of the open bosonic string spontaneous CPT violation is possible [31]. On the other hand, string theory does contain quantum field theory in certain limits, as it should. Leading-order stringy CPT-violating effects might then also be expected in the quantum-field limit. Indeed, the Standard-Model Extension (SME) mentioned below is widely utilized to capture the leading, low-energy remnants of CPT breakdown in underlying models, such as string theory.

In a more general context, we note the following. Our most fundamental models are theories for large numbers of degrees of freedom. For example, the Standard Model is essentially a many-body quantum theory. It therefore stands to reason that the underlying physics is also a theory with a large number of degrees of freedom. Past experience has shown that certain meaningful physical predictions do not require detailed knowledge of the dynamics of these degrees of freedom. For example, many features of random excitations of such systems can be described with statistical methods and thermodynamics. Such a system can also display collective excitations, in which the behavior of many degrees of freedom is correlated. Examples are water waves, phonons, or elastic deformations of macroscopic solid bodies. For the physical description of these systems at scales larger than the interatomic spacing, detailed knowledge of the motion of individual atoms is not necessary: various field-theoretic methods, including the Navier-Stokes and Beltrami-Michell equations as well as phonon quantum field theory, have proved to be successful in these contexts. These are only a few examples of a general pattern in physics: collective excitations of systems with a large number of degrees of freedom are well described by field theory in the large-distance limit. For this reason, one might expect that the dominant low-energy effects of general CPT violation arising from some unknown underlying physics are likely to be amenable to a description within the framework of ordinary quantum field theory.

Within the framework quantum field theory, there now remain two distinct options for CPT violation. These two options are highlighted by the axiomatic proof of the CPT theorem discussed in Section 2.2: Lorentz symmetry and a certain degree of smoothness in the physical laws, so that 
they remain valid for complex-valued Lorentz boosts. For CPT violation, one could therefore either give up Lorentz symmetry, or one could give up some smoothness properties of quantum field theory. This reasoning has been put on a rigorous footing by Greenberg [32]: Under the mild assumptions of the usual domain and continuity properties of the fields and energy positivity, CPT violation implies the breakdown of microcausality and Lorentz symmetry.

We begin by discussing the first option - Lorentz violation. In the last quarter century, relaxing the condition of Lorentz symmetry has developed into its own research field. This idea has been studied originally by Colladay and Kostelecký to capture the leading effects of a spontaneous breaking of Lorentz symmetry in string theory [33], as mentioned above. This approach is theoretically attractive because the mechanism of spontaneous symmetry breaking is well understood and the breaking is correspondingly benign. Moreover, the consistency of quantum field theory does not seem to require Lorentz symmetry, as the existence of many successful nonrelativistic field theories shows. This idea has led to the construction of a general field-theory framework for the description of perturbative Lorentz violation, the aforementioned SME [34-36]. In this framework, about half of the Lorentz-breaking contributions also violate CPT invariance. However, Lorentz violation without CPT breaking is certainly possible. The SME has been employed to identify, interpret, analyze, and compare numerous experimental and observational studies of Lorentz and CPT symmetry [37]. We note that at energies well below the symmetry-breaking scale, other ingredients of the CPT theorem, such as microcausality and energy positivity, are maintained in the SME [38]. The scope of this idea and the volume of experimental efforts in this context are arguably dominating the field.

A related approach has suggested the interesting possibility that a nontrivial spacetime topology evades one of the prerequisite for the CPT theorem [39]. More specifically, the case of a background Minkowski space with one of the three spatial dimensions compactified into a circle of cosmological size has been considered. This manifold continues to be a flat spacetime, avoiding potential complications due to gravity. On the other hand, the finite size of the compactified dimension imposes periodic boundary conditions, so that the plane-wave momentum spectrum is discrete along this direction. This results in a Casimir-type vacuum structure, so that $|0\rangle$ fails to be Lorentz invariant violating Axiom (2). As a result, other states in the Hilbert space violate Lorentz and CPT symmetry as well. The physical effects described by this model can be matched to the SME.

Next, we focus on the second option, giving up certain smoothness properties of physics. These smoothness properties were based on energy positivity, microcausality, the validity of closed-system quantum theory, as well as some technical assumptions, such as finite spin.

Carruthers has investigated models of bosons in which particle and antiparticle are both contained in the same isospin multiplet [40-44]. This analysis demonstrated that the conventional quantization of imposing the usual commutator relations on the creation and annihilation operators leads to non-point interactions in the case of half-integer isospin. This nonlocality, which here is known to violate microcausality, is the source of CPT violation in these models. However, multiplets of this type have not been observed in nature.

Hawking has argued that CPT violation may arise from modifications of conventional quantum mechanics due to gravitational effects $[45,46]$. The basic idea is that, in addition to the usual quantum-mechanical uncertainty principle, the gravitational field causes another fundamental limitation regarding the predictability of the future. Under ordinary circumstances, properly specified initial conditions, together with the known laws of physics, permit the prediction of observables at any later time. However, in the presence of black holes, for example, the interaction region may contain event horizons beyond which the observer has only limited physical knowledge. Part of the information about the system therefore seems inaccessible, and its quantum evolution may have to involve thermodynamic features including non-unitarity.

In addition to Hawking's idea, arguments for a different type of loss of quantum-mechanical coherence in other approaches to quantum gravity have been made, and have been reasoned to generate CPT breaking [47]. The idea is that the physics of observable low-energy particles is affected 
by unavoidable couplings to unobserved high-energy string states in the spacetime foam. This, in turn, means that the observable sector must be regarded effectively as an open quantum system leading to decoherence phenomena that include departures from CPT symmetry.

We finally mention that one of the technical requirements in Axiom (3) is that the fields have definite, finite spin. As we have illustrated, the proof of the CPT theorem with spin-zero scalar fields only, we did not need to incorporate this requirement explicitly. However, an investigation of Lorentz-symmetric infinite-spin fields by Abers, Grodsky, and Norton has revealed CPT violation as well as the presence of negative-energy states in these models [48]. Oksak and Todorov have later extended this investigation and constructed infinite-spin fields that violate CPT invariance while maintaining energy positivity [49]. These models underscore the necessity of the finite-spin requirement for $\mathrm{CPT}$ symmetry. However, particles corresponding to such fields have not been detected experimentally.

Acknowledgments: This work was supported in part by the Alexander von Humboldt Foundation, by the Indiana University Center for Spacetime Symmetries under an Indiana University Collaborative Research Grant, and by the Deutsche Forschungsgemeinschaft through Collaborative Research Center 1227 (DQ-mat).

Conflicts of Interest: The author declares no conflict of interest.

\section{References and Notes}

1. Sakharov, A.D. Pisma Zh. Eksp. Teor. Fiz. 1967, 5, 32; JETP Lett. 1967, 5, 24; Sov. Phys. Usp. 1991, 34, 392; Usp. Fiz. Nauk 1991, 161, 61.

2. Bertolami, O.; Colladay, D.; Kostelecký, V.A.; Potting, R. CPT violation and baryogenesis. Phys. Lett. B 1997, 395, 178-183.

3. Bell, J.S. Time reversal in field theory. Proc. R. Soc. Lond. 1955, 231, 479-495.

4. Lüders, G. Kgl. Danske Videnskab. Selskab Mat. Fys. Medd. 1954, $28,1$.

5. Lüders, G.; Dan, K. Proof of the TCP theorem. Ann. Phys. (NY) 1957, 2, 1-15.

6. Pauli, W. (Ed.) Niels Bohr and the Development of Physics; McGraw-Hill: New York, NY, USA, 1955; p. 30.

7. Schwinger, J.S. The theory of quantized fields. Phys. Rev. 1951, 82, 914-927.

8. Itzykson, C.; Zuber, J.B. Quantum Field Theory; McGraw-Hill: New York, NY, USA, 1980.

9. This is at least the case for Hermitian Lagrangians.

10. The CPT transformation for the photon field does not require Hermitian conjugation because $A_{\mu}^{\dagger}=A_{\mu}$. This is not necessarily the case for other spin-1 particles, such as the intermediate vector bosons $W_{\mu}^{ \pm}$.

11. The necessity of complex conjugation can, for instance, be established by the requirement that the basic structure of quantum theory, such as the canonical commutation relations, need to be maintained. As an example, consider the Schrödinger picture $[x, p]=i$, in which operators are time independent. Applying $T$ to this commutator gives $T[x, p] T^{-1}=T i T^{-1}$. Guided by the classical properties of $x$ and $p$ under time reversal, we need $T x T^{-1}=x$ and $T p T^{-1}=-p$, so that the left-hand side of the time-reversed commutator relation becomes $T[x, p] T^{-1}=[x,-p]=-[x, p]$. This results in $-[x, p]=T i T^{-1}$, which shows that we indeed must have $T i T^{-1}=-i$.

12. The connection between spin and statistics is not an independent ingredient for the CPT theorem. It follows from energy positivity and microscopic causality, as spelled out in the spin-statistics theorem. See, e.g., Fierz, M. Helv. Phys. Acta 1939, 12, 3; Pauli, W. Phys. Rev. 1940, 58, 716.

13. For products of field operators, ordering ambiguities are usually avoided by normal ordering or symmetrizing these products when constructing $\mathcal{L}$.

14. See, e.g., Streater, R.F.; Wightman, A.S. PCT, Spin and Statistics and All That; Landmarks in Mathematics and Physics, Princeton University Press: Princeton, NJ, USA, 2000.

15. Jost, R. Eine bemerkung zum CPT. Helv. Phys. Acta 1957, 30, 409.

16. For a detailed English-language version of Jost's proof, see Greenberg, O.W. Found. Phys. 2006, 36, 1535.

17. Eberhard, P.H.; Ross, R.R. Quantum field theory cannot provide faster-than-light communication. Found. Phys. 1989, 2, 127. 
18. Wightman, A.S. Quantum field theory in terms of vacuum expectation values. Phys. Rev. 1956, 101, 860.

19. Hall, D.W.; Wightman, A.S. A theorem on invariant analytic functions with applications to relativistic quantum field theory. Mat. Fys. Medd. Danske Vid. Selsk. 1957, 31, 5.

20. When these symmetry transformations are not all mutually commuting, e.g., in the case of nonabelian symmetries, they cannot all simultaneously be used to define good quantum numbers. Moreover, some care is required in certain contexts involving CPT and nonabelian global symmetries [40-44].

21. Technically speaking, this argument shows only that a particle with a given fixed momentum and helicity has the same mass as the corresponding antiparticle with the same momentum and opposite helicity. However, invoking Lorentz symmetry the mass equality can be extended to particle and antiparticle states with different momenta and to those helicities that are mixed by the Lorentz transformations. We remind the reader that helicity values may be restricted. For example, massless particles have at most two helicities regardless of the species' spin. Moreover, massless spin- $\frac{1}{2}$ particles may only come in a given helicity fixing the corresponding antiparticle's helicity to the opposite value. In particular, the existence of both helicities for such a fermion and its antifermion would be interpreted as two distinct particle species-one right-handed and its left-handed antiparticle as well as a left-handed particle and its right-handed antiparticle.

22. All spin components may need to be reversed, but this does not affect the accessible phase space resulting from energy-momentum conservation.

23. Lüders, G.; Zumino, B. Some Consequences of TCP-Invariance. Phys. Rev. 1957, 106, 385.

24. Peierls, R.E. Interpretation and properties of propagators. In Proceedings of the 1954 Glasgow Conference on Nuclear and Meson Physics, Glasgow, UK, 13-17 July 1954; Pergamon Press: New York, NY, USA, 1955.

25. Jacob, R.; Sachs, R.G. Mass and lifetime of unstable praticle. Phys. Rev. 1961, 121, 350-356.

26. Sachs, R.G. The Physics of Time Reversal; University of Chicago Press: Chicago, IL, USA, 1987.

27. Note that the electromagnetic charge quantum number $q$ represents a different concept than the electromagnetic coupling strength $e$.

28. In the special case of quantum field theory, the usual construction (37) yields a position-independent Hamiltonian. The present approach allows for the more general case of $H=H(\vec{x})$ to be position dependent.

29. In this limit, the time evolution of $H_{0}$ becomes trivial, but this does not affect the argument.

30. The correct identification of the CPT-conjugate set-up might perhaps require further theoretical input.

31. Kostelecký, V.A.; Potting, R. CPT and strings. Nucl. Phys. B 1991, 359, 545-570.

32. Greenberg, O.W. CPT Violation Implies Violation of Lorentz Invariance. Phys. Rev. Lett. 2002, 89, 231602.

33. Kostelecký, V.A.; Samuel, S. Spontaneous breaking of Lorentz symmetry in string theory. Phys. Rev. D 1989, $39,683$.

34. Colladay, D.; Kostelecký, V.A. CPT violation and the standard model. Phys. Rev. D 1997, 55, 6760.

35. Colladay, D.; Kostelecký, V.A. Lorentz-violating extension of the standard model. Phys. Rev. D 1998, $58,116002$.

36. Kostelecký, V.A. Gravity, lorentz violation, and the standard model. Phys. Rev. D 2004, 69, 105009.

37. For an overview of various tests of Lorentz symmetry see Kostelecký, V.A.; Russell, N. Rev. Mod. Phys. 2011, 83, 11. For phenomenological SME studies in this issue of Symmetry see Díaz, J.S. Symmetry 2016, 8, 105; Tasson, J. Symmetry 2016, 8.

38. Kostelecký, V.A.; Lehnert, R. Stability, causality, and Lorentz and CPT violation. Phys. Rev. D 2001, $63,065008$.

39. Klinkhamer, F.R. A CPT anomaly. Nucl. Phys. B 2000, 578, 277-289.

40. Carruthers, P. Locality and the isospin of self-conjugate bosons. Phys. Rev. Lett. 1967, 18, 353.

41. Carruthers, P. Isospin symmetry, TCP, and local field theory. Phys. Lett. B 1968, 26, 158-160.

42. Carruthers, P. Local field theory and isospin invariance. I. Free-Field theory of spinless bosons. J. Math. Phys. 1968, 9, 928-945.

43. Carruthers, P. Local Field Theory and Isospin Invariance. II. Free Field Theory of Arbitrary Spin Particles. J. Math. Phys. 1968, 9, 1835-1845.

44. Carruthers, P. Local Field Theory and Isospin Invariance. III. Interactions of Self-Conjugate Isofermion Fields. Phys. Rev. 1968, 172, 1406.

45. Hawking, S.W. Breakdown of predictability in gravitational collapse. Phys. Rev. D 1976, 14, 2460.

46. Hawking, S.W. The unpredictability of quantum gravity. Commun. Math. Phys. 1982, 87, 395-415. 
47. Ellis, J.R.; Mavromatos, N.E.; Nanopoulos, D.V. Nanopoulos. CPT Violation in string-modified quantum mechanics and the neutral-kaon system. Int. J. Mod. Phys. A 1996, 11, 1489-1507.

48. Abers, E.; Grodsky, I.T.; Norton, R.E. Diseases of infinite-component field theories. Phys. Rev. 1967, $159,1222$.

49. Oksak, A.I.; Todorov, I.T. Invalidity of TCP-theorem for infinite-component fields. Commun. Math. Phys. 1968, 11, 125-130.

(C) 2016 by the author; licensee MDPI, Basel, Switzerland. This article is an open access article distributed under the terms and conditions of the Creative Commons Attribution (CC-BY) license (http://creativecommons.org/licenses/by/4.0/). 